scheme. 30 participants were aware that parents could report using the scheme. 10 participants had been aware of an adverse drug reaction but decided not to report it. The most common reason for this was being too busy. The most common suggestion on how to improve accessibility to the Yellow Card Scheme was the implementation of a mobile phone application.

Conclusion Most participants were aware of the Yellow Card scheme although undergraduates less so. Many had reported, although some had chosen not to report because they were: too busy; not being concerned enough; not knowing how to; having forgotten. An app already exists, but awareness of this appears low, as it was the commonest suggestion to aid the low reporting.

\section{P016 PARENT/CARER INTENDED NON- ADHERENCE TO THEIR CHILD'S MEDICATION REGIMEN}

${ }^{1}$ Jeff Aston, ${ }^{2}$ Keith Wilson, ${ }^{2}$ David Terry. 'Birmingham Women's and Children's NHS Foundation Trust; ${ }^{2}$ University, Aston

10.1136/archdischild-2019-nppc.26

Aim To identify intended non-adherence reported by parents/ carers of children/young people taking long-term medication.

Methods A 10 question postal survey was sent to 180 parents of patients receiving medication via homecare at a tertiary paediatric hospital with a single repeat mailing. Demographic details collected were age, current prescribed medication and duration. Participants were asked about changes that they had made to their child's medication without consulting a healthcare professional. They were asked about delaying/not starting new medication, compliance with medication instructions, with-holding medication, altering the dose of medication, altering medication taking to fit in with daily life and strategies to aid administering medication. The data were analysed using SPSS version 23 and NVivo version 11.

Results The response rate was 32/180 (17.8\%). The mean age of respondents was 8.4 years (range 0.83 to 17 years). One hundred and fifty-eight medications were prescribed with a mean of 5 medications per patient (range 1 to 15). In total, $16 / 32(50 \%)$ respondents had made changes to their child's medication. The most common change $(9 / 32,28.1 \%)$ was adjusting the medication regimen to fit around daily life followed by delaying initiating a new medication (7/32, 20.6\%). No respondents indicated that they had not started a new prescribed medication. Six $(17.6 \%)$ respondents indicated that they had not followed the medication instructions. Four $(11.8 \%)$ respondents advised that they had withheld their child's medication. Four (11.8\%) respondents communicated that they had given a higher than prescribed dose and four $(11.8 \%)$ a lower dose. Three (8.8\%) respondents adjusted how they gave their child's medication to aid administration. Conclusion Half of respondents made changes to their child's medication without consultation with a healthcare professional. Commonly changes were made to fit around daily life. The decision to prescribe medication should be undertaken in partnership with patients. ${ }^{1}$ Adherence to medication in long-term paediatric conditions is particularly complex requiring parents to balance the daily needs of their child taking medication with everyday life. $^{2}$ Strategies to support medication adherence include self-management programmes, simplified dosing regimens and pharmacist led medication reviews. ${ }^{3}$ Parents/carers may benefit from a structured medication review for their child although further research is required to determine the effectiveness of such an intervention. This study has identified parent practices that could be included in such a review.

\section{REFERENCES}

1. National Institute for Health and Care Excellence. Medicines optimisation: the safe and effective use of medicines to enable the best possible outcomes. London: NICE. 2015.

2. Santer M, Ring N, Yardley L, et al. Treatment non-adherence in paediatric longterm medical conditions: systematic review and synthesis of qualitative studies of caregivers' views. BMC Pediatrics 2014;14:63.

3. Ryan $R$, Santesso $N$, Lowe $D$, et al. Interventions to improve safe and effective medicines use by consumers: an overview of systematic reviews. Cochrane Database of Systematic Reviews 2014, Issue 4. Art. No.: CD007768.

\section{P017 BLUE BABY BLUES - A CASE REPORT; IMPLICATIONS OF MATERNAL SELECTIVE SEROTONIN REUPTAKE INHIBITOR USE FOR SUDDEN INFANT DEATH SYNDROME}

Peter Mulholland, Alexander Simpson, Jonathan Coutts. Royal Hospital for Children

\subsection{6/archdischild-2019-nppc.27}

Background A baby girl, (38 +2 weeks, $3.026 \mathrm{~kg})$ was admitted on day 3 from home following 2 cyanotic episodes. The pregnancy was uneventful, the mother was prescribed fluoxetine $20 \mathrm{mg}$ daily during pregnancy.

Investigations Respiratory studies revealed significant hypoxia in air with episodes of hypoventilation and apnoea. Time spent below 94\% saturation was 19\%, 68 dips per hour $>4 \%, \mathrm{pCO} 2$ was raised at $7 \mathrm{kPa}$. She had a normal cranial MRI. Genetic testing for PHOX2B polyalanine expansion mutation was normal excluding Congenital Central Hypoventilation Syndrome (CCHS).

Outcome Incremental increase in the prescription of low flow oxygen normalised her saturation study. She was discharged home on day 14 with an oxygen prescription for $0.51 \mathrm{pm}$ and an apnoea monitor. Parents and family members were taught basic life support. Clinic follow up at 5 months shows baby is thriving, developing normally and the oxygen flow rate has been reduced to $0.31 \mathrm{pm}$ following repeat saturation studies.

Discussion Hypoventilation is not a recognised complication of maternal fluoxetine usage. A population based health registry study found exposure to SSRI in utero increased the rate of neonatal deaths, ${ }^{1}$ although a causal relationship could not be established. Two separate randomised controlled trials have looked at the relationship between maternal SSRI use and neonatal death. ${ }^{2}{ }^{3}$ Neither demonstrated a statistically significant correlation, although both showed odds ratios approaching statistical significance (95\% confidence intervals $0.82-1.99$ and 0.97-3.94 respectively). Mouse models demonstrate the respiratory response to acidosis is abolished by drugs targeting the serotonergic system. ${ }^{4}$ This system is not the primary regulator of respiration, ${ }^{4}$ and there may be a multi-factorial aetiology to any link between SSRI exposure in utero and the development of hypoventilation. This hypothesis somewhat correlates with the 'triple-risk model' for Sudden Unexpected Death in Infancy (SUDI), which describes three important risk factors; a critical development period, an exogenous stressor and an underlying vulnerability. It is possible that this underlying vulnerability could potentially be accounted for by downregulation of the serotonergic respiratory response in association with maternal fluoxetine use. Fluoxetine is the preferred 
SSRI for use in pregnancy. Our case has shown significant hypoventilation in an otherwise healthy infant exposed to maternal fluoxetine in utero with no primary cause identified. This potential correlation should be considered when advising mothers on safe drug use and in the management of neonatal hypoventilation.

\section{REFERENCES}

1. Colvin $\mathrm{L}$, et al. Early morbidity and mortality following in utero exposure to selective serotonin reuptake inhibitors: a population-based study in Western Australia. CNS Drugs. 2012;26:e1-14.

2. Jimenez-Solem E, Andersen JT, Petersen M, Broedbaek K, Lander AR, Afzal SA, Torp-Pedersen C, Poulsen HE. SSRI Use During Pregnancy and Risk of Stillbirth and Neonatal Mortality. American Journal of Psychiatry 2013;170:3. 299-304.

3. WuWen S, Yang Q, Garner P, Fraser W, Olatunbosun O, Nimrod C, Walker M. Selective serotonin reuptake inhibitors and adverse pregnancy outcomes. American Journal of Obstetrics and Gynaecology. 2006. 194: 4. 961-966.

4.. Voituron $\mathrm{N}$, et al. Fluoxetine Treatment Abolishes the In Vitro Respiratory Response to Acidosis in Neonatal Mice PLoS One 2010;5(10):e13644.

\section{P018 RENAL ADVERSE EVENTS AND GASTROINTESTINAL BLEEDING WITH IBUPROFEN USE IN PRETERM NEONATES WITH PATENT DUCTUS ARTERIOSUS (PDA)}

${ }^{1}$ Asma Al-Turkait, 'Janine Abramson, ${ }^{1}$ Imti Choonara, ${ }^{2}$ Lisa Szatkowski, 'Shalini Ojha. ${ }^{1}$ Child Health, Division of Graduate Entry Medicine, School of Medicine, University of Nottingham; ' Division of Epidemiology and Public Health, School of Medicine, Nottingham City Hospital

\subsection{6/archdischild-2019-nppc.28}

Aim To identify all the reported adverse events associated with ibuprofen use in preterm neonates for PDA closure and quantify the risk per 100 patients.

Methods We followed the Cochrane standards for conducting systematic reviews of adverse events. ${ }^{1}$ Eight electronic databases [Embase, Medline, BNI, PubMed, Cochrane library, IPA, CINAHL, clinical trials.gov] were searched to identify relevant studies using a predetermined search strategy. Published conference abstracts, grey literature, and reference lists of the retrieved articles were also searched. All studies providing information on adverse events of ibuprofen in preterm neonates with PDA were included. Following quality assessment of the retrieved studies, meta-analysis was performed to pool the results from the RCTs using Rev man 5.3 software. Results of the observational studies are descriptively reported and analysed. Protocol registered in PROSPERO (CRD42018067600).

Results The complete adverse events systematic review includes 84 studies (38 RCTs, 10 case reports, 4 case series, 31 cohort studies and 1 case-control study). The majority of adverse events were captured in retrospective cohort studies. Gastrointestinal (GI) bleeding: Pooled results from RCTs that compared ibuprofen to placebo showed significant difference RR [95\% CI]: $1.99[1.13,3.50]$ favouring placebo. Similarly, compared to paracetamol, ibuprofen was also associated with an increased risk of GI bleeding RR [95\% CI]: 7.00[1.91, 25.61]. There was no significant difference in GI bleeding when comparing ibuprofen to indomethacin RR [95\% CI]; 0.98[0.48, 2.00]. Renal adverse events: Data from RCTs showed that ibuprofen had a significantly low risk of oliguria compared to indomethacin RR [95\% CI]: $0.38[0.25,0.56]$. However, no difference in risk of oliguria was found when comparing ibuprofen to paracetamol RR [95\% CI]: 2.16[0.91, 5.11]. Serum creatinine levels after ibuprofen treatment compared to placebo was reported by 4 RCTs with favourable results to placebo MD [95\% CI]; 8.66 [5.17, 12.15]. The risk of adverse events per 100 patients who received ibuprofen from data from prospective studies was 8.9 for GI bleeding, 7.67.8 for oliguria, 5.2 for rise in serum creatinine and 2.6 for renal failure. Increase in serum creatinine after treatment was most commonly reported in retrospective cohort studies (460 cases out of 1786 adverse events). Nine cases of GI bleeding led to discontinuation of ibuprofen treatment.

Conclusion Our meta-analysis of the RCT data supported results of previous systematic reviews. ${ }^{2} 3$ Combined results from RCTs and prospective cohort studies in our review show that oliguria is the most commonly reported adverse event among the renal adverse events. However, the high number of rising serum creatinine after treatment from retrospective studies should also be considered when treating preterm neonates with ibuprofen for PDA. Paracetamol might be favoured as it associated with less risk of GI bleeding when compared to ibuprofen.

\section{REFERENCES}

1. Loke Y.K, Price D., Herxheimer A., et.al. Systematic reviews of adverse effects: framework for a structured approach. BMC Med Res Methodol 2007 July 5;7:32.

2. Ohlsson A., Walia R., Shah SS. Ibuprofen for the treatment of patent ductus arteriosus in preterm or low birth weight (or both) infants. Cochrane Database of Sys tematic Reviews, 2015, Issue 2. Art. No.: CD003481. doi: 10.1002/14651858. CD003481.pub6

3. Ohlsson A., Shah SS. Ibuprofen for the prevention of patent ductus arteriosus in preterm and/or low birth weight infants. Cochrane Database of Systematic Reviews, 2011, Issue 7. Art. No.: CD004213. doi:10.1002/14651858.CD004213. pub3Save

\section{P019 TREATING CONJOINED TWINS}

Lucy Wheeler. Cardiff and Vale UHB

\subsection{6/archdischild-2019-nppc.29}

Situation D and $\mathrm{M}$ are conjoined twins born without an antenatal diagnosis and assessed as not suitable for separation. At the time of admission they were 21 months old with a combined weight of $17.1 \mathrm{Kg}$. D presented unwell with a raised heart rate and respiratory rate. A working diagnosis of sepsis (possibly urinary tract infection) was made. Advice was sought from pharmacy on the doses of ceftriaxone and paracetamol. Peripheral intravenous (IV) access was only available in twin $\mathrm{M}$.

Background The twins are joined side by side from the upper chest to the pelvis. They have separate heads, three arms and 2 legs. They have 2 hearts with a fused aorta, a shared liver, 2 gallbladders, 2 stomachs, 3 kidneys and a single bladder. D has a complex congenital heart condition and a poor prognosis. On admission, D was receiving propranolol, but $M$ was not. The dose was based on the combined weight of the twins divided by 2 . Conjoined twins are a rare phenomenon, occurring 1 in 50,000 to 100,000 births. ${ }^{1}$ Around $60 \%$ of these are stillborn or die shortly after birth. There are many different types of join with differences in shared organs and limbs. Consequently each twin pair is almost unique and consideration must be given as to how medication is dosed according to pharmacokinetic principles.

Outcome Opinion of the multidisciplinary team was that the twins have relatively separate circulations, although some cross-circulation would be expected. On admission, saturations in the right arm (twin D) were 75\%. On the left side (twin M) this was 95\%. Ceftriaxone is a highly protein bound, hydrophilic antibiotic, ${ }^{2}$ The degree of cross circulation (how 